\title{
EL CRÉDITO COMERCIAL EN EL SECTOR TURÍSTICO: CICLO ECONÓMICO Y FACTORES DETERMINANTES
}

\author{
Francisco-Javier Canto-Cuevas ${ }^{1}$ \\ Profesor Asociado Facultad de CC Económicas y Empresariales (Universidad de Sevilla) \\ fcanto1@us.es \\ María-José Palacín-Sánchez ${ }^{2}$ \\ Profesora Titular Facultad de CC Económicas y Empresariales (Universidad de Sevilla) \\ palacin@us.es \\ Filippo di Pietro ${ }^{3}$ \\ Profesor Ayudante Doctor Facultad de CC Económicas y Empresariales (Universidad de Sevilla) \\ fdi@us.es
}

(Fecha envío: 09/11/16 - Fecha aceptación: 21/12/16)

\begin{abstract}
Resumen
Este trabajo estudia los factores determinantes del crédito comercial recibido y concedido en las pequeñas y medianas empresas del sector turístico español, prestando una especial atención a la situación económica. Para ello, se analiza una muestra de empresas utilizando la metodología de datos de panel durante el periodo 2004 - 2011, que incluye una etapa de expansión y otra de recesión. Los resultados muestran que el ciclo económico afecta significativamente al crédito comercial recibido y concedido. Ambos aumentan en la etapa de bonanza económica, y se contraen durante la crisis, afectando a la financiación de la empresa y a sus clientes.
\end{abstract}

\section{Palabras clave}

crédito comercial, sector turístico, PYME, ciclo económico

\begin{abstract}
This work studies the determinant factor of trade credit received and granted in medium-sized enterprises belonging to Spanish tourism sector, with special attention to economic situation. To do this, we analyze a sample of firms using the panel data methodology during the years 2004-2011, a period that includes one phase of expansion and another of recession. The results show that the economic cycle significantly affects trade credit received and granted. Therefore, both increase in the period of economic boom, and contract during crisis, which affects the financing of firms and its customers.
\end{abstract}

\section{Key words}

trade credit, tourism sector, SMEs, economic cycle

\section{INTRODUCCIÓN}

El sector turístico es uno de los sectores más significativos de la economía española. Por un lado, es uno de los que más aporta en la formación del PIB, alcanzando entre un 10\% y un 11\% en el periodo 2008-2012 según el Instituto Nacional de Estadística. Por otro lado, también se encuentra entre aquellos sectores con un mayor número de empresas, 283.000 aproximadamente en 2013, pequeñas y medianas empresas (PYME) en su gran mayoría ${ }^{4}$. Este tipo de empresas son las más vulnerables a la hora de conseguir financiación, lo que acentúa su dependencia de la financiación bancaria y del crédito comercial (Berger y Udell, 1998).

En consecuencia, es necesario profundizar en la problemática de la financiación de este sector, siendo nuestro objetivo el estudio del crédito comercial, que es uno de los instrumentos clásicos de la financiación de las PYMES y que surge cuando el proveedor permite a su cliente el diferimiento en el pago de una operación

\footnotetext{
${ }^{1}$ Máster en Dirección de Empresas y Licenciado en Ciencias Económicas y Empresariales por la Universidad de Sevilla.

2 Doctora en Administración y Dirección de Empresas por la Universidad de Sevilla.

${ }^{3}$ Doctor en Administración de Empresas por la Universidad de Sevilla y Máster en Finanzas por la Universidad de Bolonia (Italia).

${ }^{4}$ Fuente DIRCE 2013 (Instituto Nacional de Estadística)
} 
comercial. Además, aunque el crédito comercial tiene un uso generalizado en todos los sectores económicos en España (Palacín-Sánchez et al., 2011), se observa que el turístico es uno de los más afectados por la situación económica, como ocurrió durante la crisis vivida recientemente (González-Romo, 2014).

Por consiguiente, se hace necesario conectar la situación económica con el uso del crédito comercial, como ya evidenció el trabajo seminal de Schwartz (1974). Aunque tradicionalmente se ha estudiado esta relación en un contexto de expansión económica (Herbst, 1974; Mian y Smith, 1992), en los últimos tiempos están surgiendo investigaciones que analizan el crédito comercial en PYME en el contexto de la crisis en España (Carbó et al., 2015) y en países de nuestro entorno (McGuinness y Hogan, 2014; Casey y O’Toole, 2014).

El análisis empírico utiliza una muestra de PYME españolas pertenecientes a este sector y se toma como periodo de estudio de 2004 a 2011. Este periodo es muy interesante porque los primeros años (2004-2007) están caracterizados por una etapa de crecimiento económico y los últimos (2008-2011) corresponden a una recesión económica.

En general, se puede afirmar que existe escasez de estudios sobre el crédito comercial en PYME del sector turístico. Siguiendo la línea de investigación del trabajo de Canto-Cuevas et al. (2016b), que analiza el crédito comercial recibido y concedido en PYME españolas pertenecientes a todos los sectores, este artículo pretende analizar los factores determinantes y los efectos de la situación económica, tanto en expansión como en recesión, sobre el crédito comercial recibido y concedido en el caso particular de las PYME españolas del sector turístico.

Un estudio de este tipo está justificado, en primer lugar, porque es conveniente analizar cómo afecta la situación económica, y en particular la crisis, al crédito comercial de las PYME del sector turístico español, y en su doble vertiente. Es decir, como recurso financiero recibido de proveedores y como crédito concedido a clientes, que forma parte de la política comercial de la empresa. En segundo lugar, hay que tener presente las características diferenciales de la situación financiera española, que en la etapa de bonanza ha ido acompañada de una fuerte expansión crediticia y en la recesión de una sequía de crédito muy aguda que pudo afectar al crédito comercial. En Europa, el sector turístico ha incrementado su financiación comercial por las restricciones de otros recursos financieros, bancos incluidos (Casey y O'Toole, 2014). Por último, España muestra una problemática especial en cuanto al crédito comercial, situándose dentro de los países europeos con mayores plazos de pago. Alcanzando una media de 100 días durante el periodo 2008-2011 (según European Payment Index 2011 de Intrum Justitia), que indican un gran uso de dicho recurso.

Nuestros resultados muestran que la situación económica afecta a las dos vertientes del crédito comercial (proveedores y clientes) en las PYME del sector estudiado, indicando un aumento en expansión y una contracción en recesión. Además, con respecto a los factores determinantes destacar que el crédito comercial y el bancario tienen una relación negativa y por tanto sustitutiva.

El resto del artículo está organizado de la siguiente manera. En la Sección 2, de acuerdo con la teoría y evidencia empírica, se define la relación entre el crédito comercial y factores determinantes. La Sección 3 presenta los datos, variables, análisis descriptivo y metodología de nuestra investigación. A continuación, la sección 4 ofrece los resultados del análisis empírico, mientras que la Sección 5 presenta las principales conclusiones.

\section{REVISIÓN DE LA LITERATURA: TEORÍA Y EVIDENCIA EMPÍRICA}

En este apartado presentamos las relaciones esperadas entre el crédito comercial recibido y concedido con sus factores determinantes clásicos, según los estudios realizados con anterioridad.

\subsection{Factores determinantes del crédito comercial recibido}

Situación económica. Uno de los principales factores relacionados con el crédito comercial es el macroeconómico (Schwartz, 1974), debido a la influencia que tiene en la financiación empresarial en general. Nosotros esperamos que en épocas de bonanza económica las PYME hagan más uso del crédito comercial recibido y concedido, lo que evidenciaría un carácter cíclico de dicho crédito. Esto se debe, en primer lugar, a que probablemente vendan más al ser mayor su capacidad de crecimiento. En segundo lugar, a unos mayores periodos de pago y cobro (incluso una mayor relajación en condiciones de acceso al mismo). En último lugar, por el menor riesgo percibido y las mayores facilidades para acceder a todo tipo de financiación que luego se podrá trasladar a los clientes (Niskanen y Niskanen, 2006; García-Teruel y Martínez-Solano, 2010a y 2010b). En periodos de recesión, el carácter cíclico implica que el decrecimiento económico va acompañado de un descenso del crédito comercial, debido a la escasa disposición de proveedores a financiar a sus clientes por un incremento del riesgo de impago de los mismos y al endurecimiento en sus propias condiciones de financiación (Kestens et al., 2012; McGuinness y Hogan, 2014). 
Financiación bancaria. El conocimiento e influencia que el proveedor posee de su cliente permite que adopten el rol de prestamistas, actuando el crédito comercial como fuente financiera sustitutiva del crédito bancario (Meltzer, 1960; Biais y Gollier, 1997; García-Teruel y Martínez Solano, 2010a y 2010b).

Tamaño. Este factor se puede tomar como un variable proxy de la calidad crediticia de la empresa siguiendo la teoría de la información asimétrica. Por tanto, esperamos una relación positiva, según la cual, las empresas más grandes reciben más crédito de sus proveedores (Petersen y Rajan, 1997; García-Teruel y Martínez-Solano, 2010a).

Edad. Esta relación se define de manera similar a la ya establecida para el factor tamaño. Sin embargo, según la teoría del ciclo vital de las empresas (Berger y Udell, 1998), aquellas firmas que son más jóvenes y pequeñas tienden a usar más el crédito de sus proveedores (Canto-Cuevas et al., 2016a).

Autofinanciación. Relacionada con la teoría de las preferencias jerárquicas "pecking order theory" de Myers y Maljuf (1984), una relación negativa evidencia la preferencia de la financiación interna a la externa de proveedores (Niskanen y Niskanen, 2006; García-Teruel y Martínez-Solano, 2010a y 2010b).

Crecimiento. En general, las empresas que tienen un crecimiento elevado necesitan más financiación (incluida la de proveedores) para sus nuevas inversiones y viceversa (Petersen y Rajan, 1997; Cuñat, 2007; García-Teruel y Martínez-Solano, 2010a y 2010b).

Activo corriente. Es normal que las empresas ajusten el grado de liquidez de sus inversiones con el grado de exigibilidad de sus recursos. Por tanto, las empresas con una mayor inversión en circulante se financiarán también en mayor medida con recursos a corto plazo, incluido proveedores (Niskanen y Niskanen, 2006; GarcíaTeruel y Martínez-Solano, 2010a y 2010b).

\subsection{Factores determinantes del crédito comercial concedido}

Situación económica. Este factor no solo influye en el crédito comercial recibido, sino también en el concedido a clientes (Boissay y Gropp, 2007; Love y Zaidi, 2010), lo que puede suponer una vía de transmisión de perturbaciones entre compañías, en la medida en que se produzcan retrasos e impagos de clientes. Un carácter cíclico supondría que en periodos de expansión el crédito a clientes crezca, mientras que en épocas de crisis este crédito disminuya debido a que los clientes aceptan menos crédito comercial por su elevado coste, o bien los proveedores conceden menos financiación a sus clientes por la dificultad de acceso a otros recursos financieros (García-Teruel y Martínez-Solano, 2010c; Kestens et al., 2012; McGuinness y Hogan, 2014).

Tamaño. Como indicador de la valoración crediticia, las empresas más grandes están dispuestas a conceder más crédito a sus clientes por su facilidad de acceso a otros recursos financieros externos (Petersen y Rajan, 1997; Niskanen y Niskanen, 2006; García-Teruel y Martínez-Solano, 2010a; Love y Zaidi, 2010).

Edad. Siguiendo los mismos argumentos utilizados para el tamaño, aquellas empresas con más edad están dispuestas a conceder más crédito a sus clientes (Petersen y Rajan, 1997; Niskanen y Niskanen, 2006). Por otro lado, se puede considerar que las empresas de mayor edad, con mayor reputación que las jóvenes, tampoco necesitan conceder mayor aplazamiento de cobro por estar mejor establecidas (García-Teruel y MartínezSolano, 2010a).

Autofinanciación. Es de esperar que aquellas empresas con mayor capacidad de generación de recursos internos concedan más crédito a sus clientes, cumpliendo un rol de intermediarios financieros (Bougheas et al., 2009).

Crecimiento. Esperamos una relación positiva de este factor, lo cual indica que se hace uso del crédito a clientes como estrategia competitiva para cumplir objetivos de crecimiento (Petersen y Rajan, 1997; Niskanen y Niskanen, 2006)

Financiación a corto plazo. Es de suponer que las empresas que obtienen más financiación a corto plazo tienen más capacidad para financiar el activo corriente y, más concretamente, sus deudores comerciales (Atanasova y Wilson, 2003; García-Teruel y Martínez-Solano, 2010a).

Liquidez. En general, las empresas con mayor tesorería están en una mejor situación para ofrecer crédito a sus clientes (Love et al., 2007), y viceversa (Rodríguez-Rodríguez, 2008). Por otro lado, un cierto carácter involuntario en la concesión del crédito comercial evidenciaría una relación negativa con la variable liquidez (Hernández de Cos y Hernando, 1998). 


\section{MUESTRA, VARIABLES Y METODOLOGÍA}

\subsection{Muestra}

La muestra de empresas utilizada se obtuvo de la base de datos SABI de Bureau Van Dijk (Sistema de Análisis de Balances lbéricos). Se compone de PYME españolas pertenecientes al sector turístico con código NACE que comiencen por 55 y 56 , y se les exige que cumplan los tres requisitos establecidos en la recomendación 2003/361/CE respecto a empleados, ventas y activos totales ${ }^{5}$ durante los tres primeros años del periodo estudiado. Además, se filtra que las empresas tengan valores positivos en las cuentas relacionadas con el crédito comercial (clientes y proveedores), activo no corriente y corriente, y fondos propios. Finalmente, se obtiene un panel no balanceado con un total de 916 observaciones.

Respecto al periodo de estudio, este comprende de 2004 a 2011. En estos años se aprecian dos subperiodos con características económicas muy diferentes. El primero abarca de 2004 a 2007 (ambos inclusive), marcado por el crecimiento económico; mientras el segundo, de 2008 a 2011, se caracteriza por un periodo de crisis de profundidad y duración desconocidas en etapas precedentes.

\subsection{Variables}

Con respecto a las variables dependientes, por un lado, el crédito comercial recibido (PROACT) se mide como el saldo de la cuenta de proveedores dividido entre los activos totales de la empresa (Petersen y Rajan, 1997; Cuñat, 2007; García-Teruel y Martínez-Solano, 2010a y 2010b). Por otro lado, el crédito comercial concedido (CLIACT) se define como el cociente entre la cuenta de clientes y los activos totales (Kestens et al., 2012). Se han escalado ambas variables del crédito comercial por el activo total para poder recoger la importancia de dicho crédito en empresas de diferentes tamaños.

Por lo que se refiere a la variable independiente que representa la situación económica (VARPIB), la definimos utilizando la tasa de crecimiento del PIB que es un indicador básico de la situación económica de un país (Hernández de Cos y Hernándo, 1998; Niskanen y Niskanen, 2006; García-Teruel y Martínez-Solano, 2010b), y la obtenemos del Instituto Nacional de Estadística.

El estudio de la financiación bancaria la dividimos en dos variables en función de la madurez de la misma, debido a que algunas empresas pueden financiar las inversiones a corto plazo con deuda a largo plazo o puede haber un traspaso entre ambas fuentes. La primera (FINBANCP) es la ratio de deuda bancaria a corto plazo entre activos totales (García-Teruel y Martínez-Solano, 2010a y 2010b). La segunda (FINBANLP) es la ratio de deuda financiera a largo plazo entre activos totales (García-Teruel y Martínez-Solano, 2010a y 2010b).

El tamaño (TAM) es el logaritmo de los activos totales de la empresa (Petersen y Rajan, 1997; Niskanen y Niskanen, 2006; Cuñat, 2007; García-Teruel y Martínez-Solano, 2010a). La edad (EDAD) es el logaritmo de los años de vida de la empresa (Petersen y Rajan, 1997; García-Teruel y Martínez-Solano, 2010a y 2010b; Niskanen y Niskanen, 2006). La autofinanciación (AUTOFIN1) es el cociente entre el beneficio neto y los activos totales (Petersen y Rajan, 1997; Niskanen y Niskanen, 2006). El crecimiento (CREVEN) se define como incremento porcentual de las ventas respecto al año anterior (Petersen y Rajan, 1997; Niskanen y Niskanen, 2006; GarcíaTeruel y Martínez-Solano, 2010a y 2010b). El activo corriente (ACCORR) es el cociente entre los activos corrientes y los activos totales de la empresa (Niskanen y Niskanen, 2006; García-Teruel y Martínez-Solano, 2010a y 2010b).

Por último, las variables independientes que se relacionan exclusivamente con el crédito comercial concedido se definen de la siguiente manera. La autofinanciación (AUTOFIN2) es el cociente entre el beneficio neto y las ventas (Petersen y Rajan, 1997; Niskanen y Niskanen, 2006; Bougheas et al., 2009). La financiación a corto plazo (FINCP) es la ratio de pasivos corrientes entre las ventas (García-Teruel y Martínez-Solano, 2010a). La liquidez (LIQUIDEZ) es la ratio de tesorería entre los activos totales (Kestens et al., 2012).

La tabla 1 muestra los estadísticos descriptivos de todas las variables de la muestra (media, desviación típica, máximo y mínimo). Se observa que en término medio el esfuerzo que hacen las PYME del sector turístico para financiar a sus clientes es superior al crédito obtenido de sus proveedores.

\footnotetext{
${ }^{5}$ Concretamente son seleccionadas aquellas empresas con un número de empleados comprendido entre 10 y 250 , unas ventas entre 2 y 50 millones de euros, y unos activos totales que oscilan entre 2 y 43 millones de euros.
} 


\begin{tabular}{lcccc}
\hline Variable & Media & Desviación típica & Máximo & Mínimo \\
\hline PROACT & 0.062 & 0.080 & 0.673 & 0.000 \\
CLIACT & 0.099 & 0.124 & 0.940 & 0.000 \\
VARPIB & 1.496 & 2.519 & 4.100 & -3.700 \\
FINBANCP & 0.057 & 0.059 & 0.403 & 0.000 \\
FINBANLP & 0.247 & 0.192 & 0.727 & 0.000 \\
TAM & 9.462 & 0.633 & 10.856 & 7.714 \\
EDAD & 3.125 & 0.531 & 4.585 & 1.386 \\
AUTOFIN1 & 0.029 & 0.059 & 0.353 & -0.426 \\
CREVEN & 0.031 & 0.203 & 3.145 & -0.592 \\
ACCORR & 0.229 & 0.191 & 0.944 & 0.006 \\
AUTOFIN2 & 0.020 & 0.141 & 1.514 & -0.836 \\
FINCP & 0.440 & 0.501 & 7.690 & 0.037 \\
LIQUIDEZ & 0.057 & 0.086 & 0.529 & 0.000 \\
\hline
\end{tabular}

Tabla 1: Estadísticos descriptivos de las variables dependientes e independientes

\subsection{Metodología}

Para alcanzar los objetivos de nuestra investigación estimamos una ecuación para el crédito comercial recibido y otra para el concedido, que incluyen todas las variables independientes consideradas. Para llevar a cabo las estimaciones utilizamos la metodología de datos de panel. La expresión genérica para el crédito comercial recibido es la siguiente ${ }^{6}$ :

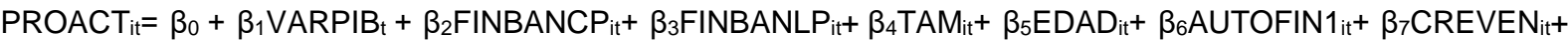
$\beta_{8} A C C O R R_{i t}++\mu_{i}+\varepsilon_{i t}$

Y para el crédito comercial concedido:

CLIACT $_{i t}=\beta_{0}+\beta_{1}$ VARPIB $_{t}+\beta_{2}$ TAM $_{i t}+\beta_{3}$ EDAD $_{i t}+\beta_{4}$ AUTOFIN2 $_{i t}+\beta_{5}$ CREVEN $_{i t}+\beta_{6}$ FINCP $_{i t}+\beta_{7}$ LIQUIDEZ $_{i t}+\mu_{i}+\varepsilon_{i t}$

Donde el subíndice i es la empresa y el $t$ es el periodo de tiempo, $\mu_{\mathrm{i}}$ representa la heterogeneidad individual inobservable y $\varepsilon_{i}$ es el término de error.

Las ecuaciones definidas son estimadas usando el modelo de efectos fijos y el modelo de efectos aleatorios para tener en cuenta los efectos individuales. Para determinar si los efectos individuales son fijos o aleatorios se realiza el test de Hausman, que permite contrastar la igualdad entre las estimaciones de efectos fijos y aleatorios. En nuestro análisis, esta prueba confirma que el modelo de efectos fijos es mejor que el modelo de efectos aleatorios.

\section{RESULTADOS}

En las tablas 2 y 3 se recogen los resultados de las regresiones de los factores determinantes del crédito comercial recibido y concedido, respectivamente. En las tablas se muestran exclusivamente los coeficientes estimados del modelo de efectos fijos, obteniendo unos resultados totalmente consistentes.

\subsection{Crédito comercial recibido}

El resultado positivo y significativo de VARPIB en las estimaciones de la tabla 2 muestra un comportamiento cíclico del crédito de proveedores con la situación económica, de modo que durante la expansión económica se produce un incremento del mismo para financiar una mayor actividad (Niskanen y Niskanen, 2006; García-Teruel y Martínez-Solano, 2010b), mientras que durante la crisis dicho crédito desciende. Esto último puede deberse a que durante las crisis los proveedores suelen endurecer las condiciones contractuales por el mayor riesgo de impago y tienen un acceso más limitado a la financiación externa (Kestens et al., 2012; McGuinness y Hogan, 2014).

\footnotetext{
${ }^{6}$ Utilizamos la expresión general de un modelo de datos de panel, aunque dicha notación se modificará en función del modelo para el análisis de datos de panel que se utilice.
} 
La relación negativa y significativa entre los factores que recogen la financiación bancaria (FINBANCP y FINBANLP) y el crédito de proveedores, evidencia una relación sustitutiva entre ambos recursos. Lo que conlleva que un descenso de la financiación bancaria se sustituya con recursos de proveedores.

Con respecto a los factores representativos de la calidad crediticia de la empresa, tamaño y edad muestran signos diferentes en su relación con el crédito de proveedores. TAM muestra un signo positivo (Petersen y Rajan, 1997; Love y Zaidi, 2010) que es indicativo de la disposición de proveedores a financiar empresas con mayores volúmenes de activos y viceversa. EDAD, con signo negativo (Niskanen y Niskanen, 2006), refleja que las empresas más jóvenes (menor reputación crediticia) acuden a la financiación de sus proveedores ante la dificultad de acceder a otros recursos.

Respecto al factor crecimiento, los resultados confirman que las PYME turísticas financian su crecimiento con proveedores y viceversa (Petersen y Rajan, 1997; Cuñat, 2007; García-Teruel y Martínez-Solano, 2010a y 2010b). La relación positiva del factor activo corriente (ACCORR) con el crédito comercial recibido refleja que las empresas con mayor inversión en circulante se financian en mayor medida con recursos de proveedores (Petersen y Rajan, 1997).

Por último, la relación negativa con la variable autofinanciación (AUTOFIN1) verifica la teoría de las preferencias jerárquicas, debido a que las empresas que generan más recursos internos se financian menos con crédito comercial.

\begin{tabular}{lrll}
\hline Variables & Efectos Fijos & \\
\hline VARPIB & 0,006 & $* * *$ & $(7,19)$ \\
FINBANCP & $-0,007^{* *}$ & $(-1,81)$ \\
FINBANLP & $-0,094^{* *}$ & $(-5,01)$ \\
TAM & $0,020^{* *}$ & $(1,79)$ \\
EDAD & $-0,0655^{* *}$ & $(-4,55)$ \\
AUTOFIN1 & $-0,1466^{* *}$ & $(-4,01)$ \\
CREVEN & $0,017^{* * *}$ & $(2,08)$ \\
ACCORR & $0,012^{* *}$ & $(0,74)$ \\
CONS & 0,096 & & $(0,87)$ \\
R-Cuadrado & $22,6 \%$ & & \\
F & 28,44 & & \\
Hausman & 6643,77 & & \\
№ de observaciones & 916 & & \\
№ de grupos & 131 & & \\
\hline
\end{tabular}

Tabla 2: Factores determinantes del crédito comercial recibido

Nota: Las estimaciones se hacen utilizando el modelo de efectos fijos. Todos los errores estándar se estiman de manera robusta con el método de Eicker/Huber/White. Entre paréntesis está el valor del estadístico t. ${ }^{* * *},{ }^{* *}$, indican significatividad al 99\%, 95\% y $90 \%$, respectivamente.

\subsection{Crédito comercial concedido}

La variable situación económica (VARPIB) presenta una relación positiva y significativa con el crédito comercial concedido en la tabla 3. Esto confirma el carácter cíclico de este crédito puesto que se incrementa en periodos de expansión debido a una mayor actividad y a una cierta relajación en las condiciones para su concesión, y disminuye durante la crisis, entre otras razones, por el endurecimiento de las condiciones de acceso al mismo. Estos resultados están en línea con la mayoría de los estudios realizados (García-Teruel y Martínez-Solano, 2010c; Kestens et al., 2012).

El hecho de que ambos créditos comerciales, recibido y concedido, se muevan en la misma dirección que la situación económica refleja cómo la cadena de suministro de la empresa, en cuyos extremos se encuentran los proveedores y los clientes, amplifica los efectos de la expansión y agrava los efectos de la crisis en cuanto a disponibilidad de crédito comercial.

Respecto a los factores de empresa tamaño (TAM) y edad (EDAD), de nuevo muestran signos diferentes que informan que las empresas más grandes y jóvenes conceden más crédito a sus clientes.

El crédito a clientes también se relaciona con factores que representan a los recursos tanto propios (AUTOFIN2) como ajenos (FINCP). En ambos casos, se relacionan positivamente con la variable dependiente, indicando que 
aquellas empresas que generan más recursos propios y tienen más recursos a corto plazo tienen más capacidad para financiar a sus clientes.

Respecto al factor crecimiento (CREVEN), los resultados sugieren que las PYME turísticas utilizan el crédito a clientes como parte de su estrategia competitiva para crecer (Petersen y Rajan, 1997; Niskanen y Niskanen, 2006). Además, el coeficiente negativo de la variable LIQUIDEZ sugiere que son las empresas con mayores dificultades en términos de tesorería las que más crédito conceden a sus clientes. Este resultado podría venir explicado por el carácter involuntario que, para algunas empresas, reviste la concesión de crédito comercial (Hernández de Cos y Hernando, 1998).

\begin{tabular}{|c|c|c|c|}
\hline Variables & Efectos Fijos & & \\
\hline VARPIB & 0,006 & $\star \star \star *$ & $(4,92)$ \\
\hline TAM & 0,022 & ** & $(1,43)$ \\
\hline EDAD & $-0,029$ & $* \star$ & $(-1,25)$ \\
\hline AUTOFIN2 & 0,058 & ** & $(2,71)$ \\
\hline CREVEN & 0,003 & ** & $(1,37)$ \\
\hline FINCP & 0,024 & ** & $(2,02)$ \\
\hline LIQUIDEZ & $-0,091$ & $* *$ & $(-2,21)$ \\
\hline CONS & $-0,038$ & & $(-0,23)$ \\
\hline R-Cuadrado & $10,3 \%$ & & \\
\hline $\mathrm{F}$ & 12,74 & & \\
\hline Hausman & 3443,77 & & \\
\hline № de observaciones & 916 & & \\
\hline № de grupos & 131 & & \\
\hline
\end{tabular}

Tabla 3: Factores determinantes del crédito comercial concedido

Nota: Ver tabla 3. ${ }^{* * *},{ }^{* *},{ }^{*}$ indican significatividad al $99 \%, 95 \%$ y $90 \%$, respectivamente.

\section{CONCLUSIONES}

En el presente artículo hemos estudiado los factores determinantes del crédito comercial concedido y recibido por las PYME españolas del sector turístico. Para ello se ha utilizado una muestra de empresas durante el periodo 2004-2011 que comprende un subperiodo de expansión y otro de crisis de características extremas.

Los resultados obtenidos para el sector turístico muestran, en primer lugar, que la situación económica afecta al crédito recibido de proveedores y al concedido a clientes de una manera cíclica. Es decir, ambos aumentan durante la expansión y disminuyen durante la crisis. Todo ello refleja que se está transmitiendo la disposición o escasez del mismo de una a otra empresa a través de la cadena de suministro. Además, esta disminución de la financiación comercial en recesión añade, si cabe, más dificultad a la falta de recursos que suele acompañar a estos periodos.

En segundo lugar, respecto al crédito comercial recibido, hay que destacar la importancia del crédito comercial como un recurso financiero usado por aquellas empresas en crecimiento. También se observa que una mayor financiación tanto interna como externa en las empresas se traduce en un menor crédito comercial recibido, predominando la relación sustitutiva entre tales recursos.

En tercer lugar, respecto al crédito comercial concedido, observamos que las PYME lo usan como herramienta competitiva, especialmente en sus años de expansión. Las empresas más jóvenes lo conceden como señal de reputación, mientras que las firmas con menor liquidez lo conceden, posiblemente, de manera involuntaria. Por otro lado, las PYME con mayor autofinanciación tienen más capacidad para ofrecer crédito a sus clientes.

Los resultados obtenidos en este artículo para el sector turístico exclusivamente, están en línea con los resultados alcanzados en el estudio de Canto-Cuevas et al. (2016b) para todos los sectores de la economía española.

Finalmente, aunque el sector turístico es un sector en el que el crédito comercial no alcanza tanta representatividad como en otros, este recurso se ve afectado por la situación económica. De tal forma que la disminución del crédito comercial concedido en épocas de recesión, podría llevar a no cumplir con las expectativas de los clientes, formados en su mayoría por grandes centrales de reservas y que podría derivar en 
malestar respecto a esta cuestión crediticia. Por lo cual, consideramos interesante en futuros estudios profundizar en la investigación de la financiación comercial respecto al tipo de clientes que componen el sector turístico.

\section{BIBLIOGRAFÍA}

Atanasova, C. V.: Wilson, N. (2003): "Bank borrowing constraint and the demand for trade credit evidence from panel data". Managerial and Decision Economics, 24: 503-514.

Berger, A. N.; Udell, G. F. (1998): "The economics of small business finance: The roles of private equity and debt markets in the financial growth cycle". Journal of Banking \& Finance, 22: 613-673.

Biais, B.; Gollier, C. (1997): "Trade credit and credit rationing”. Review of Financial Studies, 10: 903-957.

Boissay, F.; Gropp, R. (2007): "Trade credit defaults and liquidity provision by firms". Documento de trabajo del Banco Central Europeo. Vol.753.

Bougheas, S.; Mateut, S.; Mizen, P. (2009): "Corporate trade credit comercial and inventories: New evidence of a trade-off from accounts payable and receivable”. Journal of Banking \& Finance, 33: 300-307.

Canto-Cuevas, F.J.; Palacín-Sánchez, M.J.; di Pietro, F. (2016a): "Trade credit in SMEs: a quantile regression approach". Applied Economics Letters, 23 (13): 945-948.

Canto-Cuevas, F.J.; Palacín-Sánchez, M.J.; di Pietro, F. (2016b): "Efectos del ciclo económico en el crédito comercial: el caso de la pyme española". European Research on Management and Business Economics, 22: 55-62.

Carbó-Valverde, S.; Rodríguez-Fernández, F.; Udell, G.F. (2015): "Trade credit, the financial crisis, and firm access to finance". Documento de Trabajo FUNCAS, 683.

Casey, E.; O'Toole, C.M. (2014): "Bank lending constraints, trade credit and alternative financing during the financial crisis: Evidence from European SMEs". Journal of Corporate Finance, 27: 173-193.

Cuñat, V. (2007): "Trade credit: Suppliers as Debt Collectors and Insurance Providers". The Review of Financial Studies, 20: 491-527.

García-Teruel, P.J.; Martínez-Solano, P. (2010a): "Determinants of trade credit: A comparative study of European PYME”. International Small Business Journal, 28: 215-233.

García-Teruel, P. J.; Martínez-Solano, P. (2010b): "A Dynamic Perspective on the Determinants of Accounts Payable". The Review of Quantitative Finance and Accounting, 34: 439-457.

García-Teruel, P. J.; Martínez-Solano, P. (2010c): "A Dynamic Approach to Accounts Receivable: a Study of Spanish SMEs". European Financial Management, 16: 400-421.

González-Romo, Laura (2014): "Crédito comercial en España y crisis: un estudio por sectores y tamaños". Trabajo Fin de Grado, Universidad de Sevilla.

Hausman, J. (1978): "Specification Tests in Econometrics". Econometrica, 46: 1251-1271.

Herbst, A. F. (1974): "Some Empirical Evidence on the Determinants of trade credit at the Industry Level of Aggregation". The Journal of Finance and Quantitative Analysis, 9: 377-394.

Hernández de Cos, P.; Hernando, I. (1998): "El Crédito Comercial en las Empresas Manufactureras Españolas". Documento de trabajo del Banco de España, 9810.

Intrum Justitia (2011). European Payment Index. Available at: www.europeanpayment.com

Kestens, K.; Van Cauwenberge, P.; Bauwhede, H. V. (2012): "Trade credit and company performance during the 2008 financial crisis". Accounting and Finance, 52: 1125-1151.

Long, M. S.; Malitz, I. B.: Ravid, A. S. (1993): "Trade credit, Quality Guarantees, and Product Marketability". Financial Management, 22: 117-127.

Love, I.; Preve, L. A.; Sarria-Allende, V. (2007): "Trade credit and bank credit: Evidence from recent financial crises". Journal of Financial Economics, 83: 453-469.

Love, I.; Zaidi, R. (2010): “Trade credit, Bank Credit and Financial Crisis”. International Review of Finance, 10: 125-147.

McGuinness, G.; Hogan, T. (2014): "Bank credit and trade credit: Evidence from SMEs over the financial crisis". International Small Business Journal. http://dx.doi.org/10.1177/0266242614558314.

Meltzer, A. H. (1960): "Mercantile credit, monetary policy, and size of firms". Review of Economics and Statistic, 42: 429-443. 
Mian, S. L.; Smith, C. W. (1992): "Accounts receivables management policy: theory and evidence". Journal of Finance, 47: 169-200.

Myers, S. C.; Majluf, N. S. (1984): "Corporate financing and investment decisions when firms have information that investors do not have". Journal of Financial Economics, 20: 293-315.

Niskanen, J.; Niskanen, M. (2006): "The determinants of corporate trade credit policies in a bank dominated financial environment: the case of Finnish small firms". European Financial Management, 12: 81-102.

Palacín-Sánchez, M. J.; Pérez-López, C.; García-Villanueva, R. (2011): "El crédito comercial en las pymes andaluzas: oportunidad o carga". Escuela Andaluza de Economía, Sevilla.

Petersen, M. A.; Rajan, R. G. (1997): "Trade credit: theories and evidence". The Review of Financial Studies, 10: 669-691.

Rodríguez-Rodríguez, O. M. (2008): "Firms as credit suppliers: Evidence from Spanish firm-level data". International Journal of Managerial Finance, 4: 152-173.

Schwartz, R. A. (1974): "An Economic Model of trade credit". The Journal of Financial and Quantitative Analysis, 9: 643-657.

Smith, J. K. (1987): "Trade credit and informational asymmetry". Journal of Finance, 42: 863-872. 